\title{
Convergence for a Liouville equation
}

\author{
Li Ma and Juncheng C. Wei
}

\begin{abstract}
In this paper, we study the asymptotic behavior of solutions of the Dirichlet problem for the Liouville equation

$$
-\triangle u=\lambda \frac{\mathrm{K}(x) e^{u}}{\int_{\Omega} \mathrm{K}(x) e^{u}}
$$

on a bounded smooth domain $\Omega$ in the plane as $\lambda \rightarrow 8 m \pi$, where $m=1,2, \ldots$. The equation is also called the Mean Field Equation in Statistical Mechanics. By a result of H.Brezis and F.Merle, any solution sequence may have a finite number of bubbles. We give a necessary condition for the location of the bubble points.
\end{abstract}

Mathematics Subject Classification (2000). 35J60.

Keywords. Liouville equation, bubbles, asymptotic analysis.

\section{Introduction}

The Liouville equation appears in Differential Geometry and Physics. Its study has a long history. One may see [5] and [11] for discussions about physical background. Motivated by the interesting work of H.Brezis and F.Merle [3], we study the location of possible bubble points of a solution sequence when the Dirichlet boundary condition is imposed.

Let $\Omega$ be a bounded smooth domain in the plane $R^{2}$. Given a positive Lipschitz function $\mathrm{K}$ on the closure of $\Omega$, numerous authors have investigated the existence of (multiple) solutions of the Dirichlet problem of the Liouville equation on $\Omega$; namely, to find solutions $u \in \mathrm{C}^{2}(\bar{\Omega})$ satisfying

$$
\begin{gathered}
-\triangle u=\lambda \mathrm{K}(x) e^{u} / \int_{\Omega} \mathrm{K}(x) e^{u}, \quad \text { in } \Omega \\
u=0, \quad \text { on } \partial \Omega .
\end{gathered}
$$

Here $\triangle$ is the Laplacian on the plane $\mathrm{R}^{2}$ and $\lambda>0$. Note that if $u$ is a solution of (1.1-2), then by the maximum principle, we have $u>0$. Many previous results deal with the case where $\mathrm{K}=1$. In this case one has some good understanding (see [5]). In particular, when $\Omega=\mathrm{B}_{1}(0)$ and $\lambda<8 \pi$, there is a unique solution and as $\lambda \rightarrow 8 \pi^{-}$, the behavior of solution set is analyzed in [19] and [23]; while 
$\lambda \geq 8 \pi$, it is known by using a Pohozaev-type identity that (1.1-2) admits no solution at all (see [20]). However, S.S.Lin [14] (see also [5], [23] and [24] for more) proved that if $\Omega$ is a suitable annulus, there are both radial and non-radial solutions for any $\lambda>0$.

Recall that equations similar to (1.1) have been studied by many authors in the 80's (see [1] for more references) in connection with Nirenberg's problem. As in the study of Nirenberg's problem [15], there is at least one obstruction for solving (1.1-2) (see Proposition 3 below). So the geometry of the domain is crucial for the existence of solutions of (1.1-2). Some people were led to believe that for the simply-connected domain, there is no solution at all for (1.1-2) with $\lambda=8 \pi$. However, as pointed out in [5], there are regions, for example, the rectangles when the ratio between the sides is large enough, for which the concentration does not occur, so there is at least one solution for (1.1-2) with $\lambda=8 \pi$. The problem (1.1-2) also has other interesting features, which are different from other nonlinear problems like Nirenberg's problem (see [15]) and the scalar curvature problem (see [2]); in particular the blow-up analysis of H.Brezis and F.Merle [3]. In fact, for (1.12) we do have multiple bubbles [12] and when $\mathrm{K}=1$, S.Baraket and F.Pacard [26] construct them by using the fixed point theorem. By contrast, we point out that there is at most one bubble for a Palais-Smale sequence in Nirenberg's problem. Note that for problem (1.1-2) with $\lambda$ large, the Palais-Smale sequence is harder to analyze and it is still an open question. In what follows, we will write $|u|_{p}=\left(\int_{\Omega}|u|^{p}\right)^{1 / p}$ for $p \geq 1$ and let $\mathrm{G}$ be the Green function defined by

$$
-\triangle_{y} \mathrm{G}(x, y)=8 \pi \delta_{x}, y \in \Omega,
$$

and $\mathrm{G}(x, y)=0, y \in \partial \Omega$. We write $\mathrm{R}(x, y)$ the regular part of the Green function; i.e., for $x, y \in \Omega$,

$$
\mathrm{G}(x, y)=-4 \log |x-y|+\mathrm{R}(x, y) .
$$

In the following theorem we give an explicit formula for the location of the bubble points.

Theorem 1. Assume $\mathrm{K}$ is a smooth positive function on $\bar{\Omega}$. Assume $\left(u_{n}\right)$ is a blow-up solution sequence of (1.1-2) with $\lambda=\lambda_{n}$ where $\left(\lambda_{n}\right)$ converges to $8 N \pi$. Then there are finite points $\left\{x_{i}\right\}_{i=1}^{N}$ in the interior of $\Omega$ such that

$$
u_{n} \rightarrow \sum_{i} \mathrm{G}\left(., x_{i}\right), \quad \text { in } \quad \mathrm{C}_{l o c}^{2}\left(\bar{\Omega} \backslash\left\{x_{i}\right\}\right) .
$$

The set $\left\{x_{i}\right\}$ satisfies the relations

$$
\frac{1}{8 \pi} \nabla \log \mathrm{K}\left(x_{i}\right)+\nabla H_{i}\left(x_{i}\right)=0,
$$

where

$$
H_{i}(x)=\mathrm{R}\left(x, x_{i}\right)+\sum_{k \neq i} \mathrm{G}\left(x_{k}, x\right)
$$


and $i=1, \ldots, N$. In particular, for $N=1$, we have

$$
\frac{1}{8 \pi} \nabla \log \mathrm{K}\left(x_{1}\right)+\nabla H\left(x_{1}\right)=0 .
$$

Here $H(x)=\mathrm{R}\left(x, x_{1}\right)$.

The proof of this theorem is based on the moving plane method and the Pohozaev identities. The idea of using the Pohozaev identities in asymptotic analysis has been known for many years and one may see [20] for related works cited there. As a side-remark, we point out that following [10] and [28], Chen and $\mathrm{Li} \mathrm{([7]} \mathrm{and}$ [8]) used the moving plane method to obtain some beautiful apriori estimates for a class of elliptic partial differential equations in 2-dimensional domains. We remark that the special and important case when $\mathrm{K}=1$ was treated by K.Nagasaki and T.Suzuki (see [23] and [19]) using another method, which may also be applied to the case where $\mathrm{K} \neq$ constant (see [27]). It is clear that our result is also true if $\Omega$ is replaced by a compact 2-dimensional Riemannian manifold (M, $g$ ) and the equation is replaced by

$$
-\triangle_{\mathrm{M}} u=\lambda\left\{\frac{\mathrm{K}(x) e^{u}}{\int_{\mathrm{M}} \mathrm{K}(x) e^{u}}-1\right\}
$$

on $\mathrm{M}$. An interesting question may be to study the related result for the corresponding Neumann problem.

\section{Some Preparations}

We will use the blow-up analysis result of H.Brezis and F.Merle [3] for solutions of (1.1-2) with

$$
\mathrm{V}(x)=\frac{\lambda \mathrm{K}(x)}{\int_{\Omega} \mathrm{K}(x) e^{u}}
$$

in their notation. We recall their Theorem $3[3]$ and a result from Y.Y.Li and I.Shafrir [13] in the following form:

Proposition 2. Assume the function $\mathrm{V}_{n} \in \mathrm{C}^{1}(\bar{\Omega})$ satisfying

$$
0<a \leq \mathrm{V}_{n}(x) \leq b<+\infty
$$

and

$$
\left|\nabla \mathrm{V}_{n}(x)\right| \leq \mathrm{B}, \quad \text { for } \quad x \in \bar{\Omega} .
$$

Given a sequence $\left\{\xi_{n}\right\}$ of regular solutions of

$$
-\triangle \xi_{n}=\mathrm{V}_{n}(x) e^{\xi_{n}}, \quad \text { on } \Omega,
$$

with the condition

$$
\sup \int_{\Omega} \mathrm{V}_{n} e^{\xi_{n}}<+\infty
$$


Then there is a subsequence of $\left\{\xi_{n}\right\}$, still denoted by $\left\{\xi_{n}\right\}$ satisfying either (i)

$$
\left\{\xi_{n}\right\} \quad \text { is bounded in } \mathrm{L}_{\text {loc }}^{\infty}(\Omega)
$$

or (ii)

$$
\xi_{n}(x) \rightarrow-\infty \quad \text { uniformly on compact subsets of } \Omega
$$

or

(iii) there is a finite non-empty set $\mathbb{S}=\left\{a_{i}\right\}$ of $\Omega$ such that

$$
\xi_{n}(x) \rightarrow-\infty \quad \text { uniformly on compact subsets of } \Omega \backslash \mathbb{S}
$$

and for each $a_{i}$ there is a sequence $\left(x_{n}\right)$ with $x_{n} \rightarrow a_{i}$ and $\xi_{n}\left(x_{n}\right) \rightarrow+\infty$. In addition, $\mathrm{V}_{n} e^{\xi_{n}}$ converges in measure to $\sum_{i} d_{i} \delta_{i}$ with $d_{i}=8 \pi m_{i}$ where $m_{i} \geq 1$ is some integer.

We remark that the original statement of Theorem 3 in [3] is different from the form above, but their argument can be modified to cover this case.

Now we recall some well-known facts including the Pohozaev identities.

Proposition 3. Assume $\xi$ satisfies

$$
-\triangle \xi=\mathrm{V}(x) e^{\xi}, \quad \text { on } D,
$$

where $\mathrm{D}$ is a domain in $\mathrm{R}^{2}$. Then

1. Assume $\mathrm{D}$ is a closed bounded smooth domain. Then the Pohozaev identities assert that:

and

$$
\int_{\mathrm{D}} \nabla \mathrm{V}(x) e^{\xi} d x=\int_{\partial \mathrm{D}}\left(\partial_{\nu} \xi \nabla \xi-\frac{1}{2}|\nabla \xi|^{2} \nu+\mathrm{V}(x) e^{\xi} \nu\right) d \sigma
$$

$$
\begin{aligned}
& \int_{\partial \mathrm{D}}\left[(\nu \cdot \nabla \xi)(x \cdot \nabla \xi)-(x, \nu) \frac{|\nabla \xi|^{2}}{2}\right] d \sigma+\int_{\partial \mathrm{D}}(x, \nu) \mathrm{V}(x) e^{\xi} d \sigma \\
& =\int_{\mathrm{D}}(2 \mathrm{~V}+x \cdot \nabla \mathrm{V}) e^{\xi}
\end{aligned}
$$

where $\nu$ is the unit outer normal on $\partial \mathrm{D}$. We will call the last relation the original Pohozaev identity.

2. All regular solutions of $(V)$ with $\mathrm{V}=8 \pi$ and $\mathrm{D}=\mathrm{R}^{2}$ can be written as

$$
\phi_{a, \mu}(x)=\log \frac{\mu^{2}}{\left(1+\pi \mu^{2}|x-a|^{2}\right)^{2}}
$$

where $a \in \mathrm{R}^{2}$ and $\mu>0$. Here we say that $\xi$ is regular if $\xi$ satisfies the condition $\int_{\mathrm{R}^{2}} e^{\xi}<+\infty$.

The proof of the Pohozaev identities is standard and it is omitted here. As for the proof of the second part of Proposition 3 above, one may see the work of Chen and $\mathrm{Li}[8]$.

We now study the behavior of a sequence of solutions near the boundary of the domain. Taking a sequence $\lambda_{n} \rightarrow 8 m \pi$, we study the convergence of $\left(u_{n}\right)$, where $u_{n}:=u_{\lambda_{n}}$. Assume

$$
\mathrm{M}_{n}=u_{n}\left(z_{n}\right)=\max _{\bar{\Omega}} u_{n}
$$


Proposition 4. There is an uniform positive constant $d_{1}$ such that

$$
\operatorname{dist}\left(z_{n}, \partial \Omega\right) \geq d_{1}
$$

and $u_{n}$ is unformly bounded in an uniform neighborhood of the boundary of the domain.

This proposition actually can be derived from the works of Gidas, Ni, and Nirenberg [10] and De Figueiredo, Lions, and Nussbaum [28] (see [8] and [7] for related results). However, since it will play a very important role in the next section, we give a proof of it.

To prove this result, we follow the argument of [28] (see also [23]) and we need only to prove that there is an uniform constant $d_{2}>0$ depending only on $\Omega$ and $\left|\nabla\left(\log \mathrm{V}_{n}\right)\right|_{\mathrm{L}^{\infty}}$ such that for any $x_{0} \in \partial \Omega$, one has $\operatorname{dist}\left(x_{0}, z_{n}\right) \geq d_{2}$. It is clear from our assumption on $\Omega$ that $\partial \Omega$ has the uniform exterior ball property. So there is an uniform constant $r>0$ such that for every $x_{0} \in \partial \Omega$, the ball $\mathrm{B}_{r}\left(x_{1}\right) \cap \bar{\Omega}=\left\{x_{0}\right\}$ where $x_{1}=x_{0}+r \nu_{x_{0}}$ and $\nu_{x_{0}}$ is the unit outer normal vector of $\partial \Omega$ at $x_{0}$. Let $y=x-x_{1}$.

Define

$$
w_{n}(y)=u_{n}\left(x_{1}+r^{2} \frac{y}{|y|^{2}}\right)
$$

Then we have

$$
-\triangle w_{n}(y)=\frac{r^{4}}{|y|^{4}} \mathrm{~V}_{n}\left(x_{1}+r^{2} \frac{y}{|y|^{2}}\right) e^{w_{n}(y)}, \text { in } \Omega^{\prime}
$$

(we will write the right side by $\mathrm{V}^{*}\left(y, w_{n}\right)$ ) and

$$
w_{n}=0, \text { on } \partial \Omega^{\prime} .
$$

Here $\Omega^{\prime}$ is the image of the domain $\Omega$ under the conformal inversion $x \rightarrow y=$ $r^{2} \frac{x-x_{1}}{\left|x-x_{1}\right|^{2}}$. It is easy to verify that for $d>0$ small,

$$
\partial_{\nu_{0}} \mathrm{~V}^{*}(y, w) \leq 0
$$

for every $y$ in a $d$-neighborhood of $\partial \Omega^{\prime}$, where $\nu_{0}$ is the unit outer normal of $\partial \Omega^{\prime}$ at $x_{0}$. Then we can apply the argument of Theorem 2.1' (see also p.223) in [10] and conclude that there is an uniform constant $d_{2}>0$ such that there is no stationary point of $w_{n}$ (and $u_{n}$ ) in the $d_{2}$-neighborhood of $\partial \Omega^{\prime}$. Assume $\mathrm{M}_{n} \rightarrow$ $+\infty$ (otherwise we are done) and $z_{n} \rightarrow z$. Then $z$ satisfies $\operatorname{dist}(z, \partial \Omega) \geq d_{2}$. Therefore, we have the uniform apriori estimate in $\mathrm{L}^{\infty}\left(\Omega_{d_{2} / 2}\right)$ for $\left(u_{n}\right)$, where

$$
\Omega_{d_{1}}=\left\{x \in \bar{\Omega} ; \operatorname{dist}(z, \partial \Omega) \leq d_{1}\right\}
$$

In the following section, we will prove our main result. 


\section{Proof of Theorem 1}

Take a sequence of $\lambda_{n} \rightarrow 8 N \pi$ and the corresponding solution sequence $\left(u_{n}\right)$.We assume $\mathrm{M}_{n} \rightarrow \infty$. We first note, by Proposition 1, that there exist $N$-points $\left\{x_{i}\right\}$ and $N$-integers $\left\{m_{i}\right\}$ such that

$$
\mathrm{V}_{n} e^{u_{n}} \rightarrow \sum_{i} \alpha_{i} \delta_{x_{i}}
$$

weakly in measure, where $\alpha_{i}=8 \pi m_{i}$ and

$$
\mathrm{V}_{n}=\lambda_{n} \mathrm{~K} / \int_{\Omega} \mathrm{K} e^{u_{n}}
$$

Then, using Green's formula, we find that

$$
u_{n}(x)=\sum_{i} \alpha_{i} \mathrm{G}\left(x, x_{i}\right)+\int_{\Omega} \sum_{i} \alpha_{i}\left[\mathrm{G}(x, y)-\mathrm{G}\left(x, x_{i}\right)\right] \frac{\mathrm{K}(y) e^{u_{n}(y)}}{\int_{\Omega} \mathrm{K} e^{u_{n}}} d y+\circ(1) .
$$

Note, for any $r>0$, we have

$$
\sum_{i} \alpha_{i}\left[\mathrm{G}(., y)-\mathrm{G}\left(., x_{i}\right)\right] \rightarrow 0, \quad \text { in } \quad \mathrm{C}^{2}\left(\bar{\Omega} \backslash U_{i} \mathrm{~B}_{r}\left(x_{i}\right)\right)
$$

as $y \rightarrow x_{i}$. Using the convergence in measure, we obtain that

$$
u_{n}(x) \rightarrow \sum_{i} \alpha_{i} \mathrm{G}\left(x, x_{i}\right) \quad \text { on } \quad \mathrm{C}_{l o c}^{2}\left(\bar{\Omega} \backslash\left\{x_{i}\right\}\right) .
$$

For simplicity we write

$$
\overline{\mathrm{G}}(x)=\sum_{i} \alpha_{i} \mathrm{G}\left(x, x_{i}\right) .
$$

We now use the Pohozaev identities to $\xi=u_{n}$ and $\mathrm{V}=\mathrm{V}_{n}$ on the region $\mathrm{D}=\mathrm{B}_{r}:=\mathrm{B}_{r}\left(x_{1}\right)$. We get that

$$
\int_{\mathrm{B}_{r}}[\nabla \log \mathrm{K}(x)] \mathrm{V}_{n}(x) e^{u_{n}}=\int_{\partial \mathrm{B}_{r}}\left[\partial_{\nu} u_{n} \nabla u_{n}-\frac{1}{2}\left|\nabla u_{n}\right|^{2} \nu+\mathrm{V}_{n} e^{u_{n}} \nu\right] .
$$

Assume $x_{1}=0$ and we write

$$
\overline{\mathrm{G}}(x)=-4 \alpha_{1} \log |x|+H(x)
$$

where

$$
H(x)=\sum_{i \neq 1} \alpha_{i} \mathrm{G}\left(x, x_{i}\right)+\alpha_{1} \mathrm{R}(x, 0) .
$$

Notice that for $\phi \in \mathrm{C}_{0}^{1}\left(\mathrm{~B}_{r}\right)$ with $\phi=1$ on $\mathrm{B}_{r / 2}$, we have

$$
\begin{aligned}
& \int_{\mathrm{B}_{r}}[\nabla \log \mathrm{K}(x)] \mathrm{V}_{n} e^{u_{n}} \\
& =\int_{\mathrm{B}_{r}} \phi[\nabla \log \mathrm{K}(x)] \mathrm{V}_{n} e^{u_{n}}+\circ(1) \rightarrow \alpha_{1} \nabla \log \mathrm{K}(0) .
\end{aligned}
$$


In the last step we used the measure convergent of $\mathrm{V}_{n} e^{u_{n}}$. By the same argument we have

$$
\int_{\partial \mathrm{B}_{r}} \mathrm{~V}_{n} e^{u_{n}} \nu \rightarrow 0
$$

On the other hand, we have

$$
\begin{aligned}
& \int_{\partial \mathrm{B}_{r}}\left[\partial_{\nu} u_{n} \nabla u_{n}-\frac{1}{2}\left|\nabla u_{n}\right|^{2} \nu\right] \\
& \rightarrow \int_{\partial \mathrm{B}_{r}}\left[\partial_{\nu} \overline{\mathrm{G}} \nabla \overline{\mathrm{G}}-\frac{1}{2}|\nabla \overline{\mathrm{G}}|^{2} \nu\right] .
\end{aligned}
$$

Combining all these together we find

$$
\alpha_{1} \nabla \log \mathrm{K}(0)=\int_{\partial \mathrm{B}_{r}}\left[\partial_{\nu} \overline{\mathrm{G}} \nabla \overline{\mathrm{G}}-\frac{1}{2}|\nabla \overline{\mathrm{G}}|^{2} \nu\right] .
$$

In the following, we compute the integrals on the right side one by one. Without loss of generality, we assume $\alpha_{1}=8 \pi$. In fact, following the argument of Theorem 0.2 in Y.Y.Li [12] we can show by using the moving plane method that all $\alpha_{i}=$ $8 \pi$. We remark that Y.Y.Li [12] treats the general case when the domain is a compact Riemannian metric surface without boundary; however, his argument can be applied to our case because of the facts that our problem is local in nature and any Riemannian surface is locally conformal flat. To keep our presentation simple, we will not repeat it here.

Note first that, on $\partial \mathrm{B}_{r}$,

$$
\begin{aligned}
& \nu=x / r=\nabla r \\
& \nabla \overline{\mathrm{G}}(x)=-4 \frac{\nabla r}{r}+\nabla H(x),
\end{aligned}
$$

and

$$
\nabla_{\nu} \overline{\mathrm{G}}(x)=\nabla_{r} \overline{\mathrm{G}}=-\frac{4}{r}+\nabla_{r} H(x) .
$$

Then,

$$
\begin{gathered}
|\nabla \overline{\mathrm{G}}(x)|^{2}=\frac{16}{r^{2}}-\frac{8}{r} \nabla_{r} H(x)+|\nabla H(x)|^{2} \\
\nabla \overline{\mathrm{G}} \nabla_{\nu} \overline{\mathrm{G}}=\frac{16}{r^{2}} \nabla r-\frac{4}{r}\left(\nabla_{r} H \nu+\nabla H\right)+\frac{16}{r^{2}} \nabla_{r} H \nabla H,
\end{gathered}
$$

and

$$
\nabla \overline{\mathrm{G}} \nabla_{\nu} \overline{\mathrm{G}}-\frac{1}{2}|\nabla \overline{\mathrm{G}}|^{2} \nu=\frac{8}{r^{2}} \nu+\nabla H \nabla_{r} H-\frac{4}{r} \nabla H-\frac{1}{2} \nu|\nabla H|^{2} .
$$

From these, we obtain that

$$
\begin{aligned}
& \int_{\partial \mathrm{B}_{r}}\left(\nabla \overline{\mathrm{G}} \nabla \nu \overline{\mathrm{G}}-\frac{1}{2}|\nabla \overline{\mathrm{G}}|^{2} \nu\right) \\
& =-8 \pi \nabla H\left(x_{*}\right)+\circ(1),
\end{aligned}
$$

where $x_{*} \rightarrow 0$ as $r \rightarrow 0$. Here we used the fact that

$$
\int_{\partial \mathrm{B}_{r}} \nu=0
$$


Taking $r \rightarrow 0$ we find

$$
-8 \pi \nabla H(0)=\nabla \log \mathrm{K}(0)
$$

which is the desired conclusion.

\section{Acknowledgement}

The work of Ma is partially supported by the NSF of China, SRF for Returned Overseas Chinese Scholars, State Education Commission, and a scientific grant of Tsinghua University at Beijing. Ma would like to thank Prof.M.Kiessling for helpful discussions on related topics when he was in Rutgers University, New Brunswick in 1996. We are grateful to Profs.H.Brezis and M.Struwe for helpful suggestions. The authors thank the referees for the very helpful suggestions.

\section{References}

[1] Th. Aubin, Nonlinear Analysis on Manifolds, Monge-Ampere Equations. SpringerVerlag, Berlin,1982.

[2] A. Bahri, Variational problem at infinity, Pitman Math. Research Notes, 1989.

[3] H. Brezis and F. Merle, Uniform estimate and blow-up behavior for solutions of $-\triangle u=$ $\mathrm{V}(x) e^{u}$ in two dimensions, Comm. PDE. 16 (1991), 1223-1253.

[4] H. Brezis, Y. Li and I. Shafrir, A sup+inf inequality for nonlinear elliptic equations involving exponential nonlinearties, JFA 115 (1993), 344-358.

[5] E. Caglioti, P. L. Lions, C. Marchioro and M.Pulvirenti, A special class of stationary flows for two-dimensional Euler equations: A statistical mechanics description, Part 2, Comm. Math. Phys. 174 (1995), 229-260.

[6] C. C. Chen and C. S. Lin, A sharp sup-inf inequality for a nonlinear elliptic equation in the plane, Comm. Anal. and Geom. 6 (1998), 1-19.

[7] W. Chen and C. Li, A priori estimate for solutions to Nonlinear elliptic partial equations. Arch Rat. Mech. Ana. (1995).

[8] W. Chen and C. Li, Classification of solutions of some nonlinear elliptic equations, Duke J. Math. 63 (1991), 615-622.

[9] W. Ding, J. Jost, J. Li and G. Wang, The diffirential equation $\triangle u=8 \pi-8 \pi h e^{u}$ on a compact riemann surface, Asian J. Math. 1 (1997), 230-248.

[10] B. Gidas, W. M. Ni and L. Nirenberg, Symmetry and related properties via the Maximum principle, Comm. Math. Phys. 68 (1979), 209-243.

[11] M. Kiessling, Statistical mechanics of classical particles with logarithmic interaction, CPAM 46 (1993), 27-56.

[12] Y. Y. Li, Harnack type inequality: through the method of moving planes, Comm. Math.Phys. 200 (1999), 421-441.

[13] Y. Li and I. Shafrir, Blow-up analysis for solutions of $-\triangle u=\mathrm{V} e^{u}$ in dimension two, Indiana Univ. Math. J. 43 (1994), 1255-1270.

[14] S. S. Lin, On non-radially symmetric bifurcation in the annulus, J.Diff. Eq. 80 (1989), 251-279.

[15] L.Ma, Bifurcation in Nirenberg's problem, C. R. Acad. Sci. Paris, Serie I, 326 (1998), 583-588.

[16] J. Moser, On a nonlinear problem in differential geometry, Dynamical System, Acad. Press, 1973. 
[17] Moseley, Asymptotic solution for a Dirichlet problem with an exponential nonlinearity, SIAM J. Math. Analysis 14 (1983), 719-735.

[18] R. Schoen and S. T. Yau, Differential Geometry, (in Chinese), Academic Press, Beijing 1988.

[19] T. Suzuki, global analysis for two dimensional elliptic eigenvalue problems with exponential nonlinearities. Ann. I. H. P. Analyse Nonlinearie 9 (1992), 367-398.

[20] J. C. Wei, Asymptotic behavior of a nonlinear fourth order eigenvalue problem, Comm. PDE 21 (1996), 1451-1467.

[21] H.Wente, Counter-example to a conjecture of H.Hopf, Pacific J. Math. 121 (1986), 193-243.

[22] V. H. Weston, On the asymtotic solution of partial differential equation with an exponetial nonlinearity, SIAM J. Math. Analysis 9 (1978), 1030-1053.

[23] K. Nagasaki, T. Suzuki, Asymptotic analysis for two-dimensional elliptic eigenvalue problems with exponentially-dominated nonlinearities, Asymptotic Analysis 3 (1990), 173-188.

[24] K. Nagasaki, T. Suzuki, Lifting of local subdifferentiations and elliptic boundary value problems on symmetric domains II, Proc. Japan Acad. Ser. A64 (1988), 29-34.

[25] K. Nagasaki, T. Suzuki, Radial and non-radial solutions for the non-linear eigenvalue problm $\triangle u+\lambda e^{u}=0$ on the annulus in $\mathrm{R}^{2}$, J. Diff. Equations 87 (1990), 144-168.

[26] S. Baraket, F. Pacard, Construction of singular limits for a semilinear elliptic equation in dimension 2, Calc. Var. 6 (1998), 1-38.

[27] T. Senba, T. Suzuki, Some structures of solution set for a stationary system of chemotaxis, to appear in Adv. Math. Sci. Appl.

[28] D. G. De Figueiredo, P. L. Lions, R. D. Nussbaum, A priori estimates and existence of positive solutions of semilinear elliptic equations, J. Math. Pure. Appl. 61 (1982), 41-63.

Li Ma

Department of Mathematical Sciences

Tsinghua University

Beijing 100084

China

e-mail:lma@math.tsinghua.edu.cn

Juncheng C. Wei

Mathematics Department

Chinese University of Hongkong

Shatin, Hongkong

e-mail: wei@math.cuhk.edu.hk

(Received: December 3, 1999; revised version: September 10, 2000) 